Case Report

Open Access

CrossMark

\title{
A case of persistent pneumonia in a patient of ataxia telangiectasia telangiectasia
}

\section{Abstract}

Ataxia telangiectasia (AT) homozygotes have an increased risk for development of malignancies. Our patient, despite frequent courses of antibiotics, displayed persistent pulmonary infiltration, fever, and mediastinal enlarged lymph nodes on thoracic computed tomography. No infectious etiologies were established for the persistent pulmonary findings by fine needle biopsy, bronchopulmonary lavage and core lung biopsy. Histopathological examination of core lung biopsy revealed Hodgkin disease (HD).
Volume 5 Issue 8 - 2016

\section{Mohannad Mannaa}

Pediatric Pulmonology, University of Illinois College of Medicine, USA

Correspondence: Mohannad Mannaa, MD, Division Head, Pediatric Pulmonology, University of Illinois College of Medicine, USA,Emailmmannaa@uicomp.uic.edu

Received: September 10, 2016 | Published: December 07, 2016

\section{Case}

A 6-year-old boy with AT presented with a 2-week history of fever, and cough, but no shortness breath, wheezing, weight loss. His pediatrician had made the diagnosis of right lower pneumonia as proven by chest $\mathrm{x}$-ray. He was treated with azithromycin course. However, the patient did not improve and he was given a 10-day course of clindamycin in ED. The patient had no history of asthma, pneumonia, or recurrent otitis media. No history of chocking or coughing with feeding. He has an older brother with AT. He was admitted to our hospital because of persistent pneumonia and fever.

He was febrile but in no respiratory distress and no hypoxia. There were telangiectasias in the temporal and nasal parts of the conjunctivae. Chest auscultation revealed left sided crackles without wheezing. There was no evidence of clubbing or lymphadenopathy. He had mild gait ataxia. Results of a cardiac examination were negative. The rest of physical examination was unremarkable.

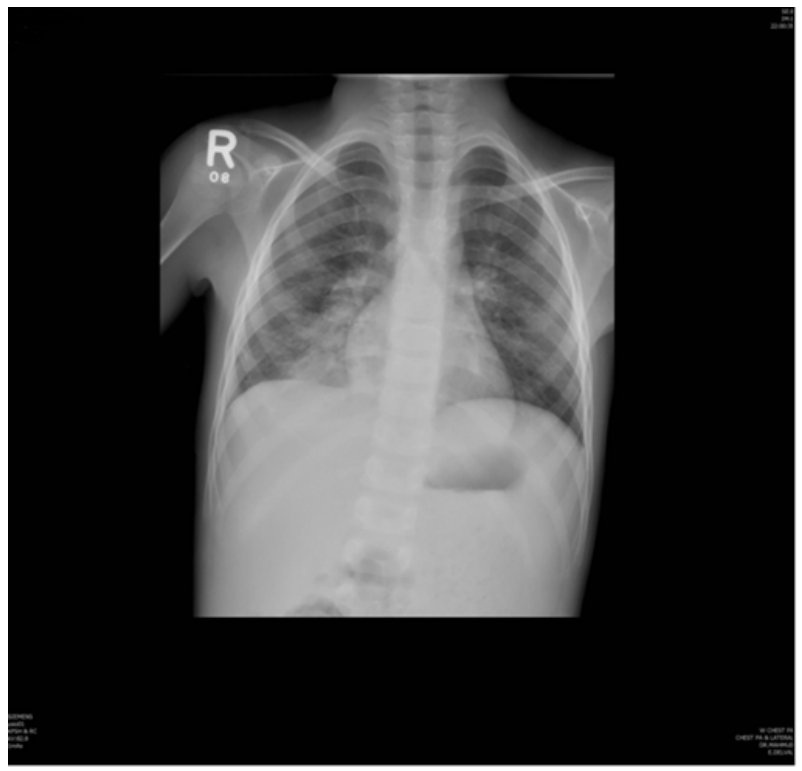

Figure I Chest radiography showed right lower and middle lobe infiltrates.

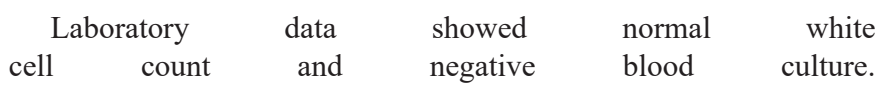

Chest radiography showed right lower and middle lobe infiltrates (Figure 1). Because of these findings our pediatric pulmonary service was consulted.

High resolution CT scan (Figure 2) of the chest showed right lower and middle lobe lung infiltrates with significant hilar lymphadenopathy. Ultrasound guided Fine needle aspiration showed no malignant cells. The patient received 2 weeks of piperacillin/tazobactam with no significant improvement so antibiotics were stopped for 3 days. Flexible bronchoscopy and core lung biopsy were scheduled. Flexible bronchoscopy and bronchoalveolar lavage (BAL) demonstrated a nonerythematous tracheobronchial tree and the histopathologic results revealed no malignant cells. Results of bacterial, viral, and fungal cultures from BAL fluid were negative. Core lung biopsy via thoracoscopy was obtained and revealed Hodgkin Lymphoma.

Patient was transferred to Oncology services for treatment.

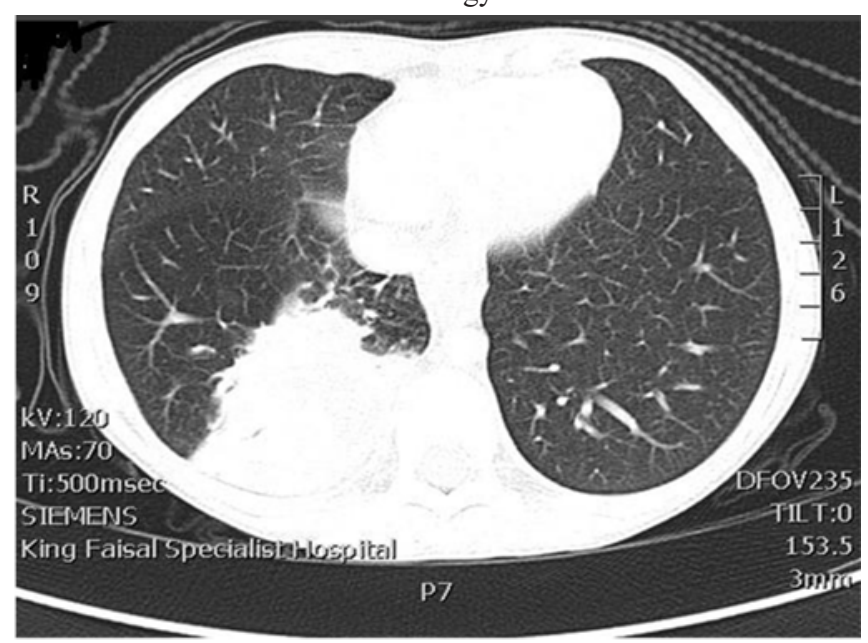

Figure 2 High resolution CT scan of the chest showed right lower and middle lobe lung infiltrates with significant hilar lymphadenopathy.

\section{Discussion}

Ataxia-telangiectasia (A-T) is clinically heterogeneous autosomal recessive progressive multisystem disease. It is characterized by progressive neurodegenerative process, cerebellar ataxia, variable immunodeficiency with susceptibility to sinopulmonary infections, 
x-ray hypersensitivity, ocular and cutaneous telangiectasia, and markedly increased predisposition to malignancy.

The clinical and immunological presentation of ataxiatelangiectasia may differ even within the same family, as described by Soresina et al. ${ }^{1}$ Most patients tend to be wheelchair bound by their second decade. Fatality occurs in the fourth or fifth decade of life. The major causes of mortality are pulmonary infections or malignancies.

Patients with AT are at additional higher risk of malignancy than individuals with any other type of primary immunodeficiency. ${ }^{2}$ The most common malignancy is Non- Hodgkin lymphoma, but other malignancies could happen such as: Hodgkin's lymphoma, leukemia, astrocytoma, medulloblastoma, gastric carcinoma, and various cutaneous neoplasms. There are few reported cases of $\mathrm{HD}$, as in our case, in A-T patients despite markedly increased risk of malignancies.

The lung involvement in AT might be caused by lung infection, interstitial lung disease due to autoimmunity and bronchiectasis secondary to recurrent infections, and abnormal lung repair as a part of AT. Recurrent sinopulmonary infections are common in AT because of the immune defect, suboptimal respiratory muscle strength, impaired airway clearance due to weak cough, swallowing dysfunction and chronic aspiration. The onset of these infections may be early in life or may be presented in 10 years or more and can lead to bronchiectasis. ${ }^{3}$
Our case showed that Hodgkin lymphoma could present as lung involvement. Malignancy should be considered when infectious cause cannot be established for persistent lung infiltrate and when there is lymphadenopathy. Patients with AT have very little lymphoid tissue, so the presence of adenopathy is highly worrisome for malignancy.

\section{Acknowledgements}

None.

\section{Conflicts of interest}

The authors declare no conflict of interest.

\section{Funding}

None.

\section{References}

1. Soresina A, Meini A, Lougaris V, et al. Different clinical and immunological presentation of ataxiatelangiectasia within the same family. Neuropediatrics. 2008;39(1):43-45.

2. Gatti RA, Good RA. Occurrence of malignancy in immunodeficiency diseases. A literature review. Cancer. 1971;28(1):89-98.

3. Buckley RH. Primary immunodeficiency diseases. In: Paul WE, editor. Fundamental immunology. Philadelphia, Lippincott-Raven. USA. 1999. p. 1427-1453. 